\title{
Curvas normales de índices de pulsatilidad y tiempo medio de desaceleración de arteria umbilical fetal en población chilena
}

\author{
Juan Carlos Bustos V. ${ }^{1}$, Vivian González C. ${ }^{1}$ \\ 1 Unidad de Perinatología, Servicio y Departamento de Obstetricia y Ginecología, Hospital San Juan de Dios, Universidad \\ de Chile.
}

\section{RESUMEN}

Objetivo: Describir la curva normal de Índices de pulsatilidad (IP) y Tiempo medio de desaceleración (t/2) en arteria umbilical fetal en población chilena. Método: Estudio transversal que incluyó: embarazo único, edad gestacional segura, una sola medición por feto, crecimiento fetal normal, embarazo normal o ausencia de patología, parto $\geq 37$ semanas. Entre las 11 y 41 semanas se realizó flujometría Doppler en arteria umbilical y se midió el IP y t/2. Resultados: Se estudiaron 877 fetos y se obtuvieron curvas de tipo polinomial para el IP $\left(y=0,0013 x^{2}-0,105 x+2,936 ; r^{2}=0,7425\right)$, se presentan los percentiles 5, 50, 90 y 95 . Para $t / 2$ se obtuvo una curva de tipo lineal $\left(y=6,4243 x+14,448 ; r^{2}=0,7749\right)$, y se presentan percentiles 5 , 10, 50 y 95 . En los exámenes bajo 20 semanas se observó que el 100\% de los fetos de 11 semanas tenían flujo umbilical ausente en diástole, proporción que disminuye hasta llegar a $0 \%$ a las 15 semanas. Conclusión: Presentamos un estudio transversal que muestra curvas de IP y t/2 de desaceleración para población Chilena. Al analizar las curvas de IP se observa una importante diferencia con las curvas de Arduini con valores entre 18 y $22 \%$ más bajos, observación que coincide con otras tablas recientes y sugiere que se deben usar estos nuevos valores para evitar subdiagnóstico de insuficiencia placentaria.

\section{PALABRAS CLAVE: Doppler umbilical, índice de pulsatilidad, tiempo medio de desaceleración}

\section{SUMMARY}

Objective: To describe a normal curve of pulsatility index (PI) and half peak systolic velocity deceleration time ( $t / 2)$ of fetal umbilical artery in chilean population. Method: Cross-sectional study including low-risk singleton pregnancy with childbirth at least 37 weeks. Doppler velocimetry was performed in umbilical artery between 11 and 41 weeks, IP and t/2 was measured. Results: 877 fetuses were studied; polynomial curves for IP were obtained $\left(y=0.0013 x^{2}-0.105 x+2.936, r^{2}=7425\right)$ are presented percentiles $5,50,90$ and 95. For $t / 2$, a linear curve was obtained $\left(y=6.4243 x+14.448 ; r^{2}=0.7749\right)$, and are presented percentiles $5,10,50$ and 95 . On examinations less than 20 weeks is observed that $100 \%$ by 11 weeks fetuses had absent umbilical diastolic flow, this proportion decreases to $0 \%$ at 15 weeks. Conclusion: We present a cross-sectional study showing curves of pulsatility index and half peak systolic deceleration time for Chilean population, analyzing IP curves observed an important difference with Arduini curves with values between 
18 and $22 \%$ lower, observation coincides with other recent tables and suggests that they should use these new values to avoid under diagnosis of placental insufficiency.

\section{KEY WORDS: Umbilical Doppler, pulsatility index, half peak systolic velocity deceleration time}

\section{INTRODUCCIÓN}

El estudio de la flujometria doppler ha sido considerado un avance muy importante en el manejo de los fetos de alto riesgo (1-7), especialmente en los casos de restricción del crecimiento intrauterino (RCIU), en los cuales el doppler de la arteria umbilical es una medida indirecta de la impedancia o resistencia placentaria (8-15).

La flujometria de la arteria umbilical se midió por primera vez con doppler con los estudios de Mac Callum y Fitzgerald $(16,17)$ en 1977 y 1978, posteriormente se desarrollaron los índices sístole / diástole (S/D), de resistencia (IR) e índice de pulsatilidad (IP), cuyos primeras tablas se publicaron hace mas de 25 años en los trabajos pioneros de Thompson y Trudinger (18); luego Harrington (19) y Arduini (20) publicaron nomogramas con un gran número de casos que son consideradas como estándar en la mayoría de los textos y centros, incluyendo las Guías Perinatales en Chile (21). Hay escasas publicaciones para reevaluar el nomograma de la arteria umbilical en la última década, también son escasas las tablas en otros países fuera de los países desarrollados, con excepción de Tailandia (22-23) y tres estudios en México y Brasil (24-26). Nuestro grupo describió un nuevo instrumento de medición de la resistencia en arteria umbilical, denominado tiempo medio de desaceleración ( $\mathrm{t} / 2$ ) aplicado inicialmente para los fetos con bradicardia, y de probable utilidad en casos de restricción de crecimiento intrauterino (27). Nos llamó la atención el escaso número de doppler alterados, aún en casos de fetos con restricción de crecimiento severo, lo que nos motivó a estudiar si las tablas de Arduini se ajustan a la normalidad de nuestras pacientes.

El objetivo de esta investigación es describir una curva normal de IP de AU entre las 11 y 41 semanas y compararla con las tablas clásicas.

\section{PACIENTES Y MÉTODOS}

Estudio prospectivo de corte transversal, se eligieron pacientes embarazadas con embarazos fisiológicos entre 11 y 41 semanas. A todas las pacientes se les realizó un examen ultrasonográfico con biometría, estimación de peso fetal, estudio anatómico y medición de velocimetria doppler en arteria umbilical, con equipo doppler color de alta resolución (Sonoace 8000 ex, Sonoace 8800 MT, Madison, Seoul, Korea; Philips HD9, Holanda), todas las pacientes dieron su consentimiento verbal.

La velocimetría Doppler se obtuvo por vía vaginal bajo 14 semanas y vía abdominal sobre esta edad gestacional, se mide en asa libre de cordón, en apnea fetal, sin movimientos fetales, con un ángulo de insonación menor a 45 grados, con un mínimo de tres ondas similares. Se eligió la onda con mayor velocidad y menos ruido, se midió índice de pulsatilidad (IP) y tiempo medio de desaceleración $(t / 2)$, si el resultado estaba alterado según tabla de Arduini (20) se informaba al médico tratante.

Los criterios de inclusión fueron: (1) embarazo único; (2) edad gestacional segura con ultrasonido de primer trimestre; (3) una sola medición por feto; (4) peso fetal estimado y/o peso de nacimiento entre los percentiles 10 y 90; (5) embarazo normal o ausencia de patología que comprometa el crecimiento fetal al momento del examen; (6) parto $\geq 37$ semanas. Se excluyeron específicamente los embarazos con malformaciones, oligohidroamnios, parto prematuro, restricción de crecimiento intrauterino, hipertensión y diabetes.

Parte de este grupo de pacientes se utilizó para otro estudio citado previamente en que se estudio el nomograma normal de tiempo medio de desaceleración entre las 17 y 41 semanas.

\section{RESULTADOS}

Se estudiaron 877 fetos, que cumplían con todos los criterios de inclusión, se obtuvieron los datos completos del parto en el $56 \%$ de las pacientes. En la Figura 1 se muestra el nomograma de distribución del IP según edad gestacional, la distribución alcanzo la mejor correlación con una curva polinomial $\left(y=0,0013 x^{2}-0,105 x+2,936\right.$; $\left.r^{2}=0,7425\right)$, se calcularon los percentiles $5,50,90$ y 95 (Tabla I). 


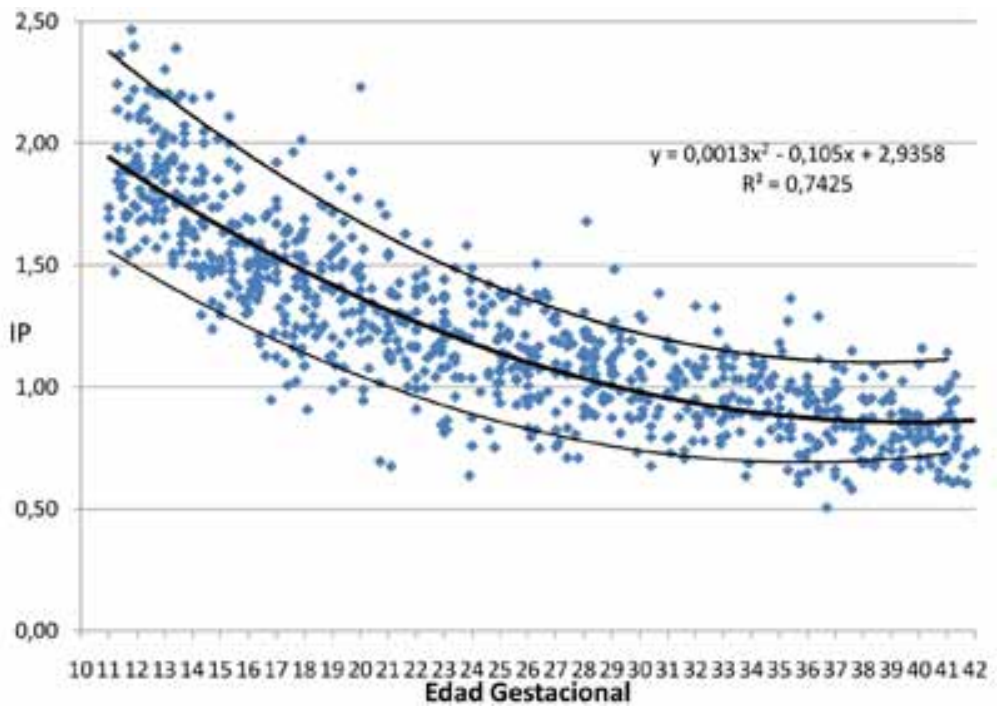

Figura 1. Índice de pulsatilidad de arteria umbilical en 877 fetos: las líneas muestran los percentiles 5, 50 y 95.

Tabla I

ÍNDICES DE PULSATILIDAD (IP) DE ARTERIA UMBILICAL EN 877 FETOS

\begin{tabular}{|c|c|c|c|c|c|}
\hline EG & $n$ & p 5 & p 50 & p 90 & p 95 \\
\hline 11 & 33 & 1,56 & 1,87 & 2,22 & 2,38 \\
\hline 12 & 45 & 1,49 & 1,80 & 2,14 & 2,28 \\
\hline 13 & 42 & 1,42 & 1,74 & 2,06 & 2,20 \\
\hline 14 & 33 & 1,36 & 1,67 & 1,99 & 2,11 \\
\hline 15 & 31 & 1,30 & 1,61 & 1,92 & 2,03 \\
\hline 16 & 34 & 1,24 & 1,56 & 1,85 & 1,95 \\
\hline 17 & 38 & 1,19 & 1,50 & 1,78 & 1,88 \\
\hline 18 & 32 & 1,14 & 1,45 & 1,72 & 1,81 \\
\hline 19 & 27 & 1,09 & 1,39 & 1,66 & 1,74 \\
\hline 20 & 27 & 1,04 & 1,35 & 1,60 & 1,68 \\
\hline 21 & 28 & 1,00 & 1,30 & 1,55 & 1,61 \\
\hline 22 & 30 & 0,96 & 1,25 & 1,50 & 1,56 \\
\hline 23 & 28 & 0,92 & 1,21 & 1,45 & 1,50 \\
\hline 24 & 21 & 0,89 & 1,17 & 1,40 & 1,45 \\
\hline 25 & 34 & 0,86 & 1,14 & 1,36 & 1,41 \\
\hline 26 & 26 & 0,83 & 1,10 & 1,32 & 1,36 \\
\hline 27 & 25 & 0,80 & 1,07 & 1,28 & 1,32 \\
\hline 28 & 33 & 0,78 & 1,04 & 1,25 & 1,28 \\
\hline 29 & 25 & 0,76 & 1,01 & 1,22 & 1,25 \\
\hline 30 & 23 & 0,74 & 0,99 & 1,19 & 1,22 \\
\hline 31 & 23 & 0,72 & 0,97 & 1,16 & 1,19 \\
\hline 32 & 22 & 0,71 & 0,95 & 1,14 & 1,17 \\
\hline 33 & 23 & 0,70 & 0,93 & 1,12 & 1,15 \\
\hline 34 & 21 & 0,70 & 0,91 & 1,10 & 1,13 \\
\hline 35 & 23 & 0,69 & 0,90 & 1,08 & 1,12 \\
\hline 36 & 29 & 0,69 & 0,89 & 1,07 & 1,11 \\
\hline 37 & 21 & 0,69 & 0,88 & 1,06 & 1,10 \\
\hline 38 & 23 & 0,70 & 0,88 & 1,05 & 1,10 \\
\hline 39 & 26 & 0,70 & 0,87 & 1,05 & 1,10 \\
\hline 40 & 28 & 0,71 & 0,87 & 1,05 & 1,11 \\
\hline 41 & 23 & 0,73 & 0,87 & 1,05 & 1,11 \\
\hline
\end{tabular}

EG: edad gestacional (semanas).
En la Figura 2 se muestra el nomograma de distribución del t/2 según edad gestacional, la distribución alcanzó la mejor correlación con una curva lineal $\left(y=6,4243 x+14,448 ; r^{2}=0,7749\right)$, se calcularon los percentiles 5, 10, 50 y 95 (Tabla II).

En los embarazos menores a 20 semanas se analizó la proporción de fetos con flujo ausente en diástole. Se encontró un $100 \%$ a las 11 semanas que disminuye a $64 \%, 41 \%, 9 \%$ a las 12,13 y 14 semanas respectivamente, no hubo casos desde las 15 semanas en adelante (Figura 3).

En la Figura 4 se compara el percentil 95 de diversas series desde 1990 hasta la actual, comparado con las tablas de Arduini (20); se eligió este percentil porque es el valor que la mayoría de los estudios recomienda como punto de corte para predecir compromiso fetal.

\section{DISCUSIÓN}

El Doppler de la arteria umbilical es una medida de la resistencia placentaria. Múltiples estudios han demostrado que los índices de resistencia disminuyen con la edad gestacional (19-23), sin embargo los nomogramas que fueron publicados a fines de los años ochenta, son aún de amplio uso en todo el mundo y recomendados en los principales textos de Ultrasonografía $(21,28)$. 


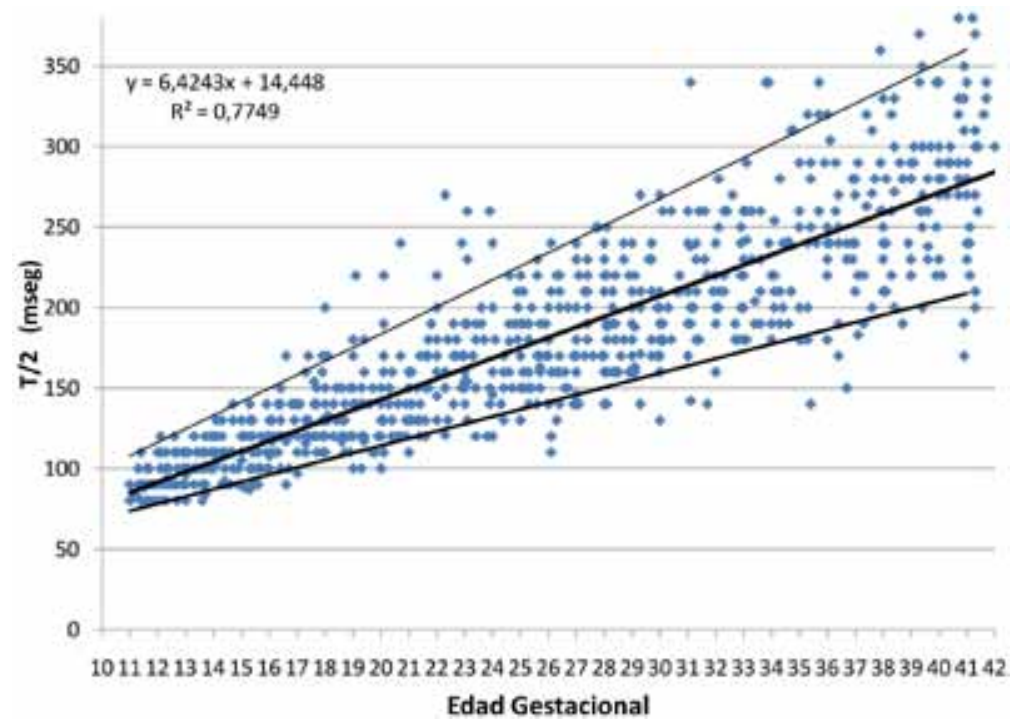

Figura 2. Tiempo medio de desaceleración en 877 fetos: las líneas muestran los percentiles 5, 50 y 95.

Tabla II

TIEMPO MEDIO EN MILISEGUNDOS DE DESACELERACIÓN EN 877 FETOS

\begin{tabular}{cccccc}
\hline EG & $\mathrm{n}$ & $\mathrm{p} \mathrm{5}$ & $\mathrm{p} 10$ & $\mathrm{p} \mathrm{50}$ & $\mathrm{p} \mathrm{95}$ \\
\hline 11 & 33 & 74 & 76 & 84 & 108 \\
12 & 45 & 78 & 80 & 90 & 116 \\
13 & 42 & 83 & 85 & 97 & 125 \\
14 & 33 & 87 & 90 & 104 & 133 \\
15 & 31 & 92 & 95 & 110 & 141 \\
16 & 34 & 96 & 100 & 117 & 150 \\
17 & 38 & 101 & 105 & 123 & 158 \\
18 & 32 & 105 & 109 & 130 & 167 \\
19 & 27 & 110 & 114 & 137 & 175 \\
20 & 27 & 114 & 119 & 143 & 184 \\
21 & 28 & 119 & 124 & 150 & 192 \\
22 & 30 & 123 & 129 & 156 & 200 \\
23 & 28 & 128 & 133 & 163 & 209 \\
24 & 21 & 132 & 138 & 170 & 217 \\
25 & 34 & 137 & 143 & 176 & 226 \\
26 & 26 & 141 & 148 & 183 & 234 \\
27 & 25 & 146 & 153 & 189 & 243 \\
28 & 33 & 150 & 158 & 196 & 251 \\
29 & 25 & 155 & 162 & 202 & 259 \\
30 & 23 & 159 & 167 & 209 & 268 \\
31 & 23 & 164 & 172 & 216 & 276 \\
32 & 22 & 168 & 177 & 222 & 285 \\
33 & 23 & 173 & 182 & 229 & 293 \\
34 & 21 & 177 & 186 & 235 & 302 \\
35 & 23 & 182 & 191 & 242 & 310 \\
36 & 29 & 186 & 196 & 249 & 318 \\
37 & 21 & 191 & 201 & 255 & 327 \\
38 & 23 & 195 & 206 & 262 & 335 \\
39 & 26 & 200 & 211 & 268 & 344 \\
40 & 28 & 204 & 215 & 275 & 352 \\
41 & 23 & 209 & 220 & 282 & 360 \\
\hline
\end{tabular}

EG: edad gestacional (semanas).
Doppler umbilical : flujo ausente en diảstole

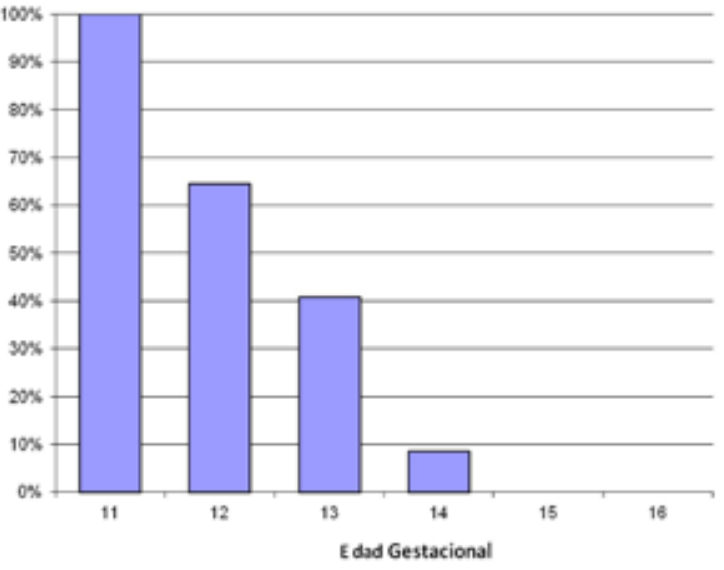

Figura 3. Porcentaje de flujo ausente en diástole en arteria umbilical.

Se han descrito diversos índices para el estudio de la flujometría doppler (18), la opinión actual es que en arteria umbilical el IP es mejor por varias razones (28): los índices S/D e IR no se pueden usar con los flujos ausente o reverso en diástole, los índices S/D e IR son similares en información del doppler espectral, ya que solo utilizan los valores extremos en sístole y diástole, incluso son transformables entre sí mediante la sencilla formula IR = 1 - (1/ [índice s/d]). EI IP entrega más información de la onda doppler ya que incluye el valor medio de la onda. 


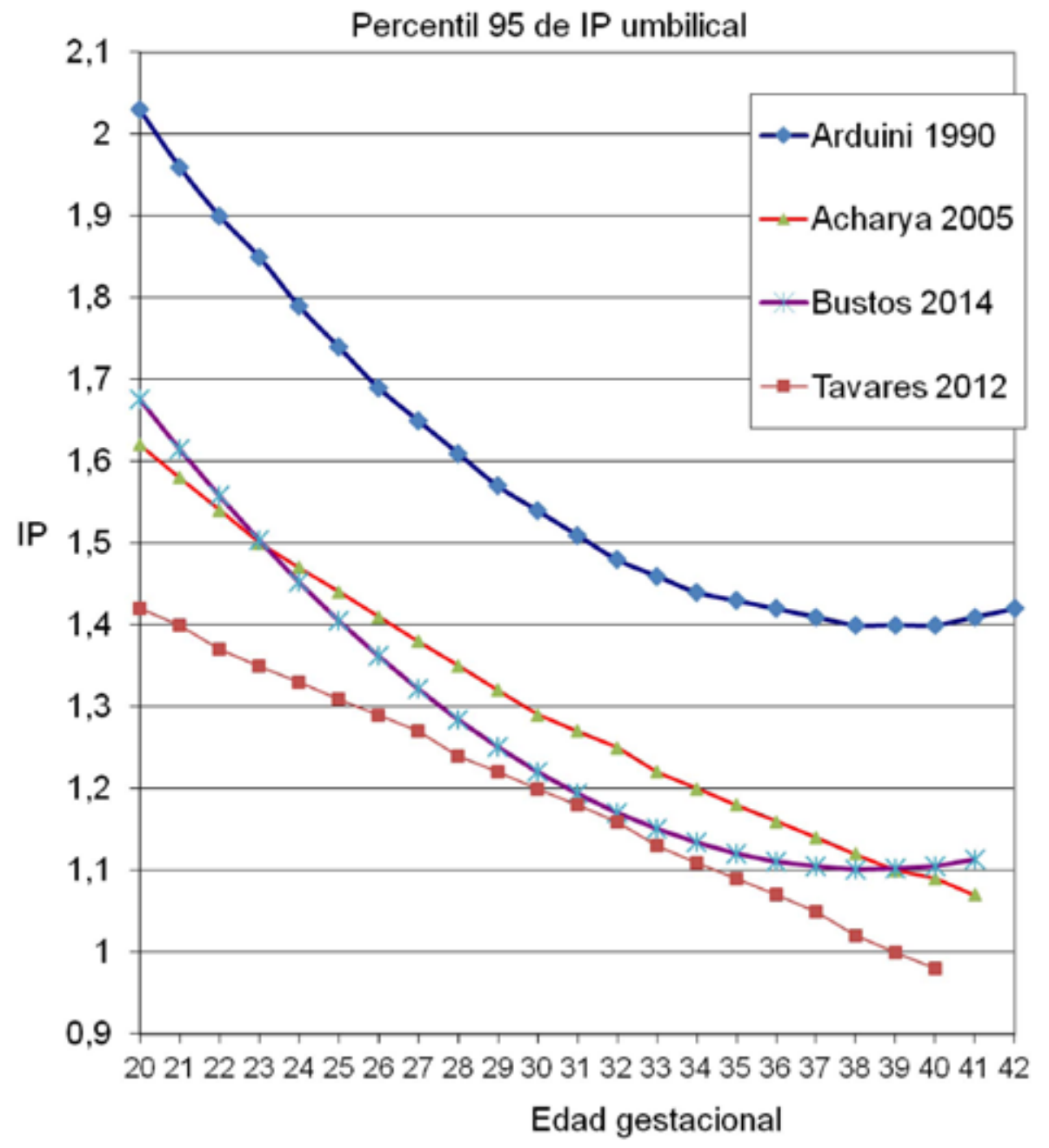

Figura 4. Comparación de percentiles 95 para diversas curvas de índice de pulsatilidad en arteria umbilical.

Recientemente nuestro grupo ha descrito un nuevo instrumento (27): el tiempo medio de desaceleración, que es realmente un índice de conductancia placentaria (inverso de la resistencia), demuestra una notable progresión lineal con el tiempo de embarazo y estudia los valores de la porción media de la onda, y que parecen alterarse precozmente en la RCIU y puede ser de especial utilidad en casos de flujo ausente o reverso, alteraciones de la frecuencia cardiaca fetal y gemelares. La mayoría de las curvas comparten la forma general con una disminución continua del índice de pulsatilidad con la edad gestacional y un leve aplanamiento de la curva en las últimas semanas de gestación (Figura 4).
Este estudio es la primera tabla descrita en Chile y una de las pocas en América Latina. En la bibliografía se ha publicado la tabla de Medina en 2006 realizada en España y México (25); otro estudio de Parra y cols (29) publica tablas pero con pacientes de Gran Bretaña. Un estudio reciente de Tavares en Brasil en 2012 (26) es longitudinal y con buena casuística y metodología, sin embargo, mide el cordón cerca de la placenta y del abdomen fetal, a diferencia del resto de los estudios que mide en asa libre.

Las curvas de IP de Arduini (20) y Harrington (19) construidos en la década de los 90, muestran valores claramente más elevados que los de Acharya (30), Tavares (26) y los nuestros construi- 
dos en esta década. La diferencia de los valores con la tabla de Arduini de 1990 es significativa; cuando comparamos el percentil 95 nuestros fetos muestran valores de IP entre 0,3-0,35 puntos (18$22 \%$ ) menores a los de Arduini; solo dos valores de los $592(0,34 \%)$ fetos sobre 20 semanas de nuestro grupo están sobre el p95 de Arduini. Esta diferencia también fue notada por Medina (25), quien especula que puede ser debida a la mejoría tecnológica y de técnica de medición por ej. la mejor captación de las ondas doppler de baja velocidad y el aumento en la frecuencia de repetición de pulsos, también se puede argüir diferencias de población o metodológicas.

Nuestra tabla demuestra valores muy similares a los de Acharya (30), este es un estudio mixto transversal y longitudinal con 513 observaciones de 130 pacientes realizada en pacientes noruegas en 2004. El autor no excluye aquellos casos de embarazadas que luego del examen hacen alguna patología del embarazo con el fin de evitar una curva de "supernormales". El estudio de Tavares (26) en Brasil es longitudinal, entre quienes se excluyeron las pacientes que desarrollaron patologías después de su inclusión, además que no excluía a fetos grandes para la edad gestacional, por lo cual podría calificar como una curva de "supernormales" y explicar que sus valores sean inferiores a los de Acharya y los nuestros.

Una consecuencia importante de la diferencia de valores con la tabla de Arduini (20) que es ampliamente utilizada en el mundo y específicamente recomendada en Chile por la Guía Perinatal, es que esta tabla pudiera subdiagnosticar casos de compromiso fetal inicial, dicho de otra manera cierto porcentaje de fetos serían normales para Arduini pero alterados según la nueva tabla, en el grafico estos valores estarían en el "callejón" entre ambos percentiles 95 (Figura 4).

Un aporte original de nuestro estudio es que publicamos valores desde las 11 semanas y permite evaluar resistencia placentaria desde antes de las 20 semanas de la tabla de Arduini; la utilidad de estos valores precoces debe ser establecida: hay algunas observaciones que la resistencia placentaria puede estar alterada precozmente en fetos con aneuploidias (a partir del segundo trimestre) y en casos de restricción de crecimiento severo (a partir de las $18-20$ semanas) $(31,32)$. Es notable que a las 11 semanas de gestación todos los fetos tengan flujo ausente en diástole, luego la resistencia placentaria cae rápidamente y nuestro estudio demuestra que desde las 15 semanas la ausencia de flujo en diástole es patológica.

\section{CONCLUSIÓN}

Presentamos un estudio transversal que muestra curvas de índice de pulsatilidad y tiempo medio de desaceleración para población chilena. Al analizar las curvas de IP se observa una importante diferencia con las curvas de Arduini con valores entre 18 y $22 \%$ más bajos, lo que sugiere que se deben usar estos valores para evitar subdiagnóstico de insuficiencia placentaria.

\section{REFERENCIAS}

1. American College of Obstetricians and Gynecologists. ACOG Practice Bulletin no. 134: Fetal growth restriction. Obstet Gynecol 2013;121(5):1122-33.

2. Figueras F, Gardosi J. Intrauterine growth restriction: new concepts in antenatal surveillance, diagnosis, and management. Am J Obstet Gynecol 2011;204(4):288300.

3. Pilliod RA, Cheng YW, Snowden JM, Doss AE, Caughey $A B$. The risk of intrauterine fetal death in the small-for-gestational-age fetus. Am J Obstet Gynecol 2012;207(4):318.e1-6.

4. Caughey $A B$. How best to diagnose and treat the small-for-gestational-age fetus. Am J Obstet Gynecol 2013;209(5):397-9.

5. Unterscheider J, O'Donoghue K, Daly S, Geary MP, Kennelly MM, McAuliffe FM, Hunter A, Morrison JJ, Burke G, Dicker P, Tully EC, Malone FD. Fetal growth restriction and the risk of perinatal mortality-case studies from the multicenter PORTO study. BMC Pregnancy Childbirth 2014;14(1):63.

6. Baschat AA, Cosmi E, Bilardo CM, Wolf $\mathrm{H}$, et al. Predictors of neonatal outcome in early-onset placental dysfunction. Obstet Gynecol 2007;109(2 Pt 1):253-61.

7. Resnik R. Intrauterine growth restriction. Obstet Gynecol 2002;99(3):490-6.

8. Rasmussen S, Irgens LM. History of fetal growth restriction is more strongly associated with severe rather than milder pregnancy-induced hypertension. Hypertension 2008;51(4):1231-8.

9. Warrington JP, George EM, Palei AC, Spradley FT, Granger JP. Recent advances in the understanding of the pathophysiology of preeclampsia. Hypertension 2013;62(4):666-73.

10. American College of Obstetricians and Gynecologists; Task Force on Hypertension in Pregnancy. Hypertension in pregnancy. Report of the American College of Obstetricians and Gynecologists' Task Force on Hypertension in Pregnancy. Obstet Gynecol 2013;122(5):1122-31.

11. Long PA, Abell DA, Beischer NA. Fetal growth retardation and pre-eclampsia. Br J Obstet Gynaecol 1980;87(1):13-8.

12. Lees $\mathrm{C}$, Marlow N, Arabin B, Bilardo CM, Brezinka $C$, et al. Perinatal morbidity and mortality in earlyonset fetal growth restriction: cohort outcomes of the trial of randomized umbilical and fetal flow in 
Europe (TRUFFLE). Ultrasound Obstet Gynecol 2013;42(4):400-8.

13. Alfirevic Z, Neilson JP. Doppler ultrasonography in high-risk pregnancies: systematic review with metaanalysis. Am J Obstet Gynecol 1995;172(5):1379-87.

14. Scifres CM, Stamilio D, Macones GA, Odibo AO. Predicting perinatal mortality in preterm intrauterine growth restriction. Am J Perinatol 2009; 26(10):723-8.

15. Baschat AA, Gembruch U, Reiss I, Gortner L, Weiner CP, Harman CR. Relationship between arterial and venous Doppler and perinatal outcome in fetal growth restriction. Ultrasound Obstet Gynecol 2000;16(5):407-13.

16. FitzGerald DE, Drumm JE. Non-invasive measurement of human fetal circulation using ultrasound: a new method. Br Med J 1977;2(6100):1450-1.

17. McCallum WD, Williams CS, Napel S, Dígale RE: Fetal Blood velocity waveforms. Am J Obstet Gynecol 1978;132:425-7.

18. Thompson RS, Trudinger BJ, Cook CM. Doppler ultrasound waveform indices: $A / B$ ratio, pulsatility index and Pourcelot ratio. $\mathrm{Br} \mathrm{J}$ Obstet Gynaecol 1988;95(6):581-8.

19. Harrington K, Carpenter RG, Nguyen M, Campbell S. Changes observed in Doppler studies of the fetal circulation in pregnancies complicated by pre-eclampsia or the delivery of a small-for-gestational-age baby. I. Cross-sectional analysis. Ultrasound Obstet Gynecol 1995;6(1):19-28.

20. Arduini D, Rizzo G. Normal values of Pulsatility Index from fetal vessels: a cross-sectional study on 1556 healthy fetuses. J Perinat Med 1990;18(3):165-72.

21. González R, Nien JK, Carstens M, Medina L. Vigilancia antenatal. En: Guía Perinatal. 1aㅡ Edición. CEDIP. Santiago de Chile. Ministerio de Salud. 2003. pp 5570.

22. Sunsaneevithayakul $P$, Siwadune $T$, Boriboonhirunsarn $D$, et als. Reference centile charts for fetal umbilical arteries doppler velocimetry waveform of Thai fetuses. Thai J Obstet Gynaecol 2000;12:201-11.

23. Sutantawiboon A, Chawanpaiboon $S$. Doppler study of umbilical artery in Thai fetus. J Med Assoc Thai 2011;94(11):1283-7.
24. Rodriguez Ballesteros R, López Sánchez ME, Chapa Fernández JG, Galván González JL, Olivares Morales AS. Normal distribution curve for umbilical arterial flow velocimetry (S/D relation) measured by Doppler ultrasonography. Ginecol Obstet Mex 1993;61:171-5.

25. Medina N, Figueroa H, Guzmán M., Hernández E. Valores de referencia del índice de pulsatilidad de las arterias uterina y umbilical durante el embarazo. Ginecol Obstet Mex 2006:74:509-15.

26. Tavares NM, Ferreira SG, Bennini JR, Marussi EF, Barini R, Peralta CF . Longitudinal reference intervals of maternal-fetal Doppler parameters. Rev Bras Ginecol Obstet 2013;35(1):33-8.

27. Bustos JC, Paublo M, Ramírez P, Sepúlveda W. Umbilical artery half peak systolic velocity deceleration time throughout pregnancy and its role in fetuses with bradycardia. Ultrasound Obstet Gynecol 2007;30(7):952-7.

28. Figueras F, Meler E, Gómez O. Vasos e índices de la exploración básica. En: Doppler en medicina fetal. Gratacós E. (Ed) 1aㅡ edición. Madrid. Ed Médica Panamericana. 2010. pp 35-48.

29. Parra-Cordero M, Lees C, Missfelder-Lobos H, Seed $P$, Harris C. Fetal arterial and venous Doppler pulsatility index and time averaged velocity ranges. Prenat Diagn 2007;27:1251-7.

30. Acharya G, Wilsgaard T, Berntsen G, Maltau J, Kiserud $T$. Reference ranges for serial measurements of umbilical artery Doppler indices in the second half of pregnancy. Am Journal Obstet Gynecol 2005;192(3):937-44.

31. Bustos JC, González V, Sepúlveda W. Umbilical artery half peak systolic velocity deceleration time in fetal growth restriction and perinatal death. Presentado como poster en 12th World Congress in Fetal Medicine, Junio 2013. Marbella. España.

32. Bustos JC, González V, Sepúlveda W, Bustos-Orellana JC. Umbilical artery pulsatility index and half peak systolic velocity deceleration time in Down syndrome. Presentado como poster en 12th World Congress in Fetal Medicine, Junio 2013. Marbella. España. 